\title{
Statistical characteristics of finger-tapping data in Huntington's disease
}

\author{
Chrystalina A. Antoniades · Jan Ober • \\ Stephen Hicks - Gill Siuda • R. H. S. Carpenter • \\ Christopher Kennard • Andrea H. Nemeth
}

Received: 14 July 2011 / Accepted: 3 January 2012/Published online: 19 January 2012

(C) The Author(s) 2012. This article is published with open access at Springerlink.com

\begin{abstract}
Measuring the rate of finger tapping is a technique commonly used as an indicator of impairment in degenerative neurological conditions, such as Huntington's disease. The information it provides can be greatly enhanced by analysing not simply the overall tapping rate, but also the statistical characteristics of the individual times between each successive response. Recent technological improvements in the recording equipment allow the responses to be analysed extremely quickly, and permit modification of the task in the interest of greater clinical specificity. Here we illustrate its use with some pilot data from a group of manifest HD patients and age-matched controls. Even in this small cohort, differences in the responses are apparent that appear to relate to the severity of the disease as measured by conventional behavioural tests.
\end{abstract}

C. A. Antoniades $(\bowtie) \cdot$ S. Hicks · C. Kennard · A. H. Nemeth Division of Clinical Neurology, Nuffield Department of Clinical Neurosciences, Level 6 West Wing, John Radcliffe Hospital, University of Oxford, Oxford OX3 9DU, UK

e-mail: Chrystalina.antoniades@clneuro.ox.ac.uk

C. A. Antoniades - R. H. S. Carpenter

Department of Physiology, Development and Neuroscience, University of Cambridge, Downing Street,

Cambridge CB2 3EG, UK

J. Ober

Maciej Nalecz Institute of Biocybernetics and Biomedical

Engineering, Polish Academy of Sciences, Warsaw, Poland

G. Siuda · A. H. Nemeth

Dementia and Neurodegenerative Diseases Network,

John Radcliffe Hospital, Oxford, UK

A. H. Nemeth

Department of Clinical Genetics, Churchill Hospital,

Oxford, UK
Keywords Finger tapping · Huntington's disease

\section{Introduction}

Huntington's disease (HD) is a devastating inherited neurodegenerative condition. It is characterised not only by the movement abnormalities, but also by cognitive impairment and abnormal behaviour, as well as weight problems and sleep disturbances [9]. HD patients experience a complex variety of movement problems, which include not only chorea, but also akinesia and bradykinesia. A number of studies have assessed impairment of simple motion sequences in HD patients as a way of quantifying the progression of symptoms $[2,7,12,15]$.

Sequential hand movements in HD patients have been examined at various stages of the condition [17], with markedly slower execution of movements by the HD patients when compared with controls. As well as performing movements more slowly, HD patients are also slower in switching from one movement to the next [1]. Garcia Ruiz and colleagues [7] studied the degree of bradykinesia and timing in genetically confirmed HD individuals compared with controls, using the four CAPIT timed tasks previously used for PD [10]. There were no significant differences between patients with and without anti-dopaminergic drugs. These results have been reproduced in more recent studies that reported significantly reduced tapping rates in manifest HD patients as compared to controls, but not premanifest individuals; Unified Huntington's Disease Rating Scale (UHDRS) motor scores and duration of the disease were highly correlated with the tapping results, something which did not correlate with the CAG repeat lengths [3, 13-15].

Longitudinal studies have shown a significant decline in tapping rate over a period of 3 years in manifest HD 
patients, and a strong correlation between UHDRS scores and the motor tests [2]. Furthermore, the variability of finger tapping (using target intervals of 600 and 1,200 ms) correlated with an index of the probability of motor onset, estimated from CAG length and age [8].

Quick and easy-to-use hand tapping devices have enabled the number of taps in $30 \mathrm{~s}$, and the variability in tapping rhythm and fatigue over the testing period, to be measured. Initial cross-sectional testing of HD patients using an early model of such a device showed that the tapping frequency correlated significantly with the motor UHDRS and independence scores [11]. Longitudinal data from a small cohort followed over 10 years revealed that this correlation was maintained over time, suggesting the technique may provide an objective measure of disease progression. Recently, a large study, TrackHD, used a force transducer to measure paced finger tapping in premanifest and manifest HD patients; significant differences between premanifest and manifest patients were found, again suggesting that finger-tapping measures are an important way of monitoring the progression of the disorder [16].

Here we present a pilot study, using a new portable device in which the sensors are activated purely by contact and are independent of force; this facilitates the rapid collection of finger-tapping data, and allows the possibility of introducing modifications of the basic task that may have diagnostic utility. Because it provides information not merely about average tapping rates, but about the statistical distribution of the individual intervals between responses, it enables the behaviour to be characterised more specifically and quantitatively, sometimes revealing aberrant patterns of response that would not be detected by conventional average measures; this is likely to aid monitoring and diagnosis.

\section{Materials and methods}

\subsection{Participants}

The present study was approved by the Local Regional Ethics Committee and was conducted in the John Radcliffe Hospital, Oxford, UK. Participants were recruited from the patients attending the regional HD clinic. All had a positive genetic test for HD, and were evaluated by an experienced clinician (AHN) using a standard neurological examination and the UHDRS to ascertain whether individuals had motor manifestation of Huntington's disease (Table 1). There were two experimental groups: eight in a manifest group (MG) with overt motor signs, and a small aged-matched group of three controls (C). All participants gave their informed consent after the procedures had been explained to them.
Table 1 Characterisation of the manifest (M) patient group and controls $(\mathrm{C})$

\begin{tabular}{lll}
\hline & $\mathrm{M}(n=8)$ & $\mathrm{C}(n=3)$ \\
\hline Age: mean \pm SD & $54.50 \pm 10.08$ & $54.33 \pm 7.81$ \\
CAG repeats & $42.17 \pm 1.80$ & N/A \\
UHDRS (motor score) & $31.75(11-81)$ & N/A \\
$\quad$ (mean, range) & $12.00 \pm 0.71$ & N/A \\
Total functional capacity & $26.86(17-45)$ & N/A \\
VF (mean, range) & & \\
Hand tapping & & \\
$\mu$ (reciprocal median latency) & & \\
mean \pm SE (s $\left.{ }^{-1}\right)$ & $4.08 \pm 0.60$ & $4.95 \pm 0.85$ \\
$\quad$ Single & $3.18 \pm 0.41$ & $4.06 \pm 0.53$ \\
$\quad$ Alternating & $2.14 \pm 0.30$ & $3.49 \pm 0.74$ \\
Pronating & & \\
$\sigma$ (SD of main distribution) & & $0.35 \pm 0.12$ \\
mean \pm SE (s ${ }^{-1}$ ) & & $0.44 \pm 0.21$ \\
$\quad$ Single & $0.75 \pm 0.14$ & $0.41 \pm 0.07$ \\
$\quad$ Alternating & $0.49 \pm 0.06$ & \\
Pronating & $0.41 \pm 0.03$ & \\
\hline
\end{tabular}

Except where stated, all figures are mean $\pm 1 \mathrm{SE}$

\subsection{Recording the finger tapping}

The tapping pad (Fig. 1 left: Ober Consulting Poland) has two touch sensors each of $60-\mathrm{mm}$ diameter, their centres 115-mm apart; each has an associated blue LED providing feedback whenever one of the sensor active fields is touched. The sensors are activated purely by contact, independent of force, as they work by monitoring electrical impedance, with a threshold that is automatically adjusted to the background noise level. Left and right taps are recorded independently, at a sampling frequency of $1 \mathrm{kHz}$ (resolution $1 \mathrm{~ms}$ ), for a fixed period of 30s. Participants sat in front of the pad with their forearm resting on the table. Three protocols were used, and in each case the subject used their dominant hand: (1) alternation: the instructions were to tap the left and right sensors alternately as rapidly as possible using the tip of the index finger; (2) pronation/supination: alternately as before and as rapidly as possible, but with the hand in the supine position when tapping medially side, but prone on when tapping laterally, using the finger nail rather than finger tip; (3) single: tapping the right sensor only, with the index finger. For each task, the successive intervals between taps were recorded, generating histograms of the distribution of intervals between consecutive taps. The statistics of these inter-tap intervals for hand tapping was then analysed using the computer application SPIC (saccadic programming and instrumentation computer [4]), calculating best-fit parameters ( $\mu$ and $\sigma$ ) of the LATER (linear approach to threshold with ergodic rate) model [5]. 


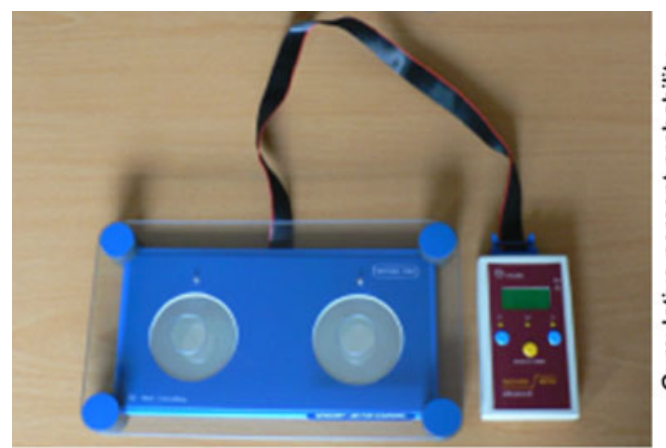

Fig. 1 Left The portable device for recording hand tapping. The subject taps on the circular sensor areas. Right A typical reciprobit plot of hand-tapping interval data from one subject in this study tapping alternately on the two sides: responses for right and left

\section{Results}

\subsection{Distributions of inter-tap intervals}

Figure 1 (right) shows a typical distribution plot from one of the participants in the study. As usually found for saccadic and evoked manual reaction times, distributions of the intervals between taps in this task are skewed, with a long tail of longer latencies, and in general the reciprocal of reaction time, or promptness, follows a Gaussian distribution (and is therefore more amenable to statistical analysis).

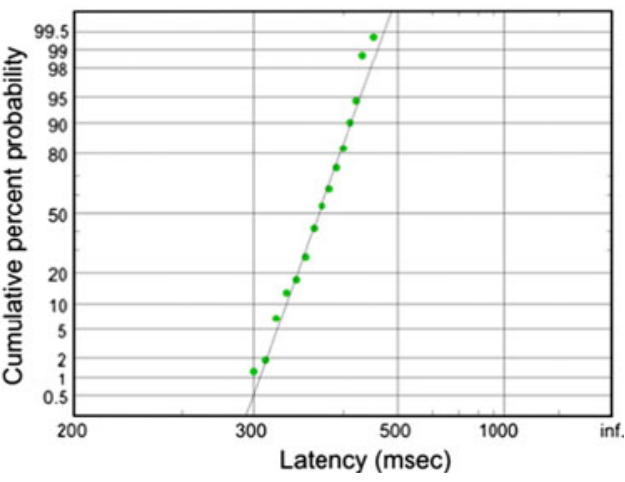

movements are combined. The points represent a cumulative histogram of the latencies (intervals between taps), plotted on a probability scale as a function of reciprocal latency

Consequently if inter-tap interval distributions are plotted cumulatively, on a probit scale, using a reciprocal abscissa (a reciprobit plot), they will be expected to generate a straight line, as seen on Fig. 1 (right). Such a distribution can be fully described by just two parameters: these are $\mu$, its mean (which is also the reciprocal of the median latency), and $\sigma$, its standard deviation. A large value of $\mu$ corresponds to increased promptness or speed of response, and thus a shorter interval; because of the reciprocal relationship, the units for these two parameters are $\mathrm{s}^{-1}$ or Hz. The best-fit values of the parameters can be determined automatically
Fig. 2 Examples of unusual latency distributions. a A subject showing marked bimodality in the alternation task, with one group of responses around $300 \mathrm{~ms}$ and another around $450 \mathrm{~ms}$ : responses in each direction are very similar. b A subject showing sporadic inattention in the pronation task: in both directions a small group of responses (top right) is markedly delayed relative to the main distribution. c Marked left/ right difference; responses to the right are about $40 \mathrm{~ms}$ faster (alternation task). d A manifest HD patient with exceptionally slow responses, affecting all three types of movement, but most marked for the pronation/ supination task

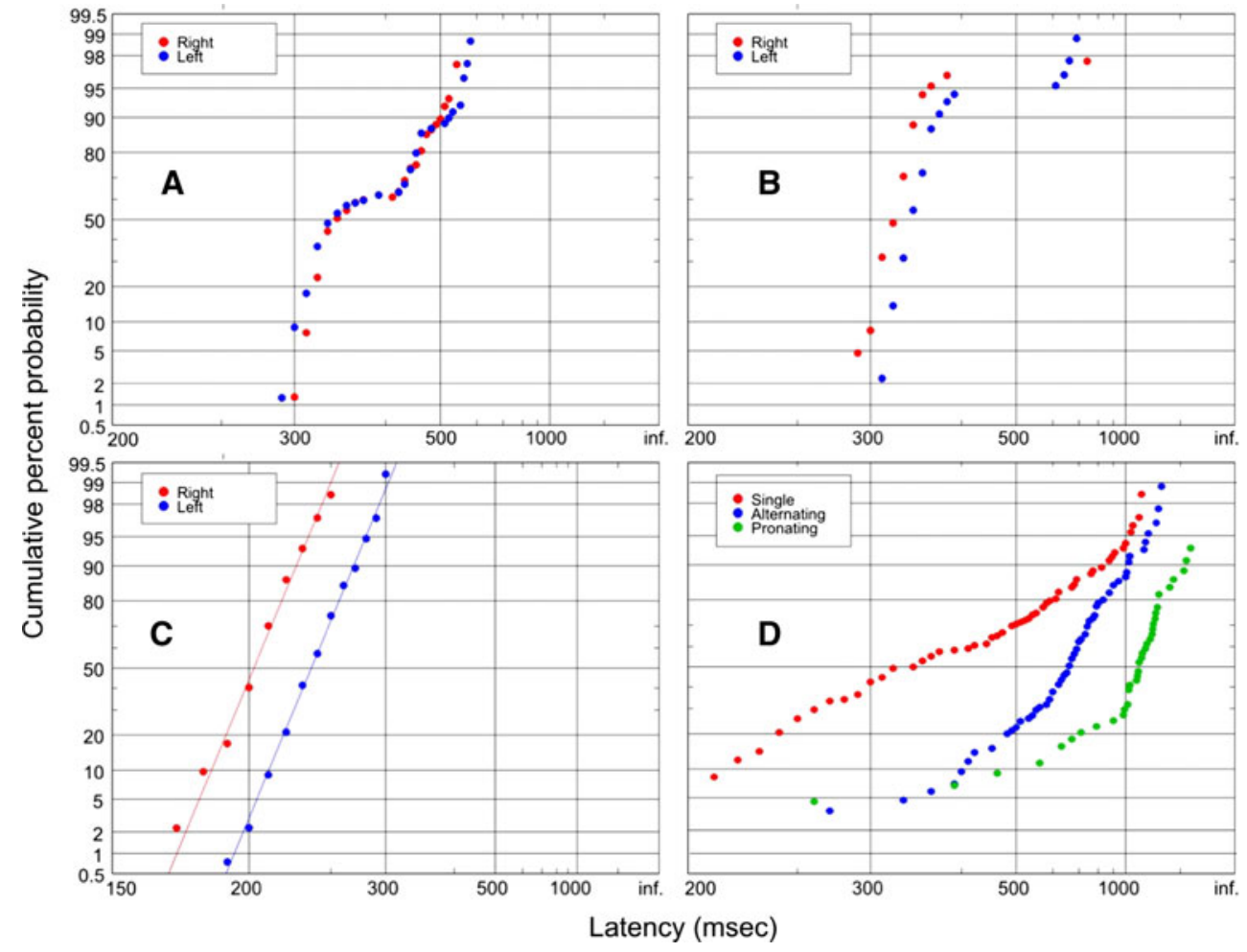


Fig. 3 Average values of $\mu$ (left) and $\sigma$ (right) analysed by type of response and category of participant $(C$ control, $M$ manifest HD). Error bars show 1SE
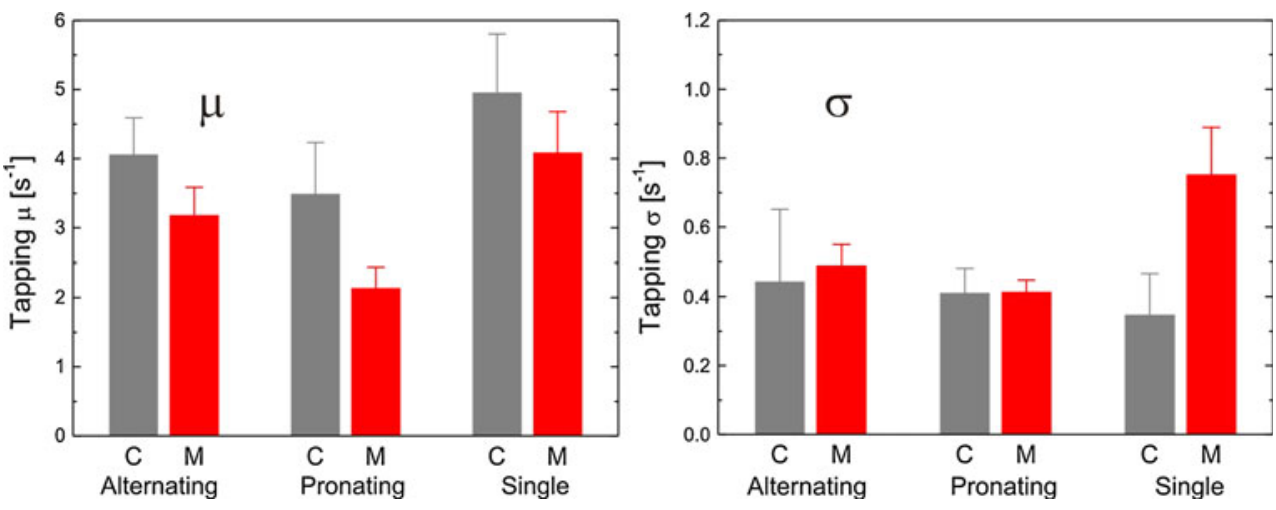

by minimisation of the Kolmogorov-Smirnov one-sample statistic.

For most subjects, the observed distribution of reciprocal inter-tap intervals has the expected normal distribution, but in some cases significant, idiosyncratic deviations are observed. Figure 2 shows examples of unusual distributions for individual participants in this study. Figure $2 \mathrm{a}$ demonstrates a bimodal pattern of response, possibly analogous to the population of 'express' saccades sometimes seen in saccadic latency distributions, first described by Fischer [6]. A different kinds of abnormality (Fig. 2b) is the existence of a distinct sub-set of aberrantly long intertap intervals (around 600-700 ms), probably due to inattention: in this case they represent only some $5 \%$ of all trials. Some participants show marked differences for rightward and leftward movements (Fig. 2c), implying some asymmetry of neurological function. Finally, Fig. 2d shows responses from one manifest HD patient with remarkably long inter-tap intervals, and very marked

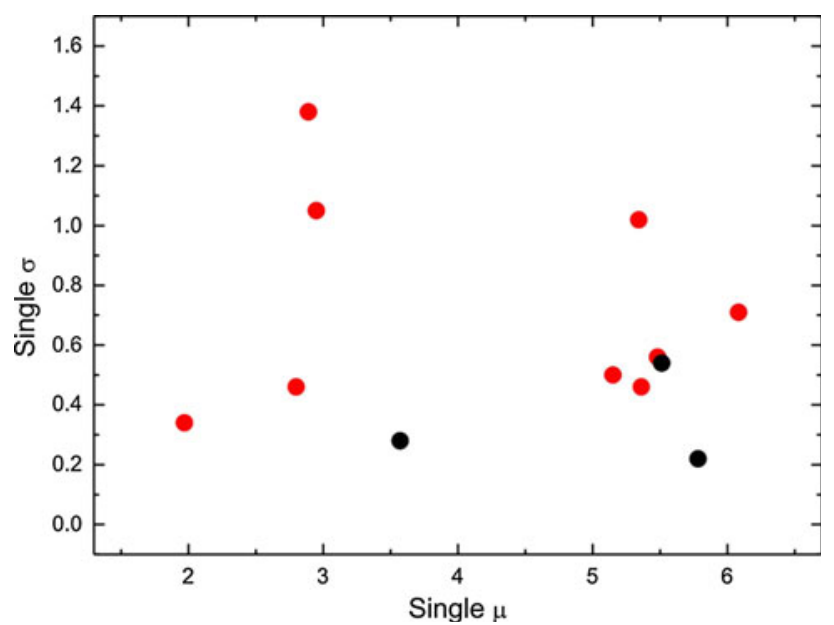

Fig. 4 Scatter plot of $\sigma$ versus $\mu$ for the two groups of participants performing single tapping; despite the very small numbers in this pilot study, the manifest HD patients ( $r e d$ ) seem to form a distinct cluster from the controls (black) differences between the three types of task. An important point to note is that many of these characteristic features would have been entirely missed by the conventional technique of simply counting the total number of taps in a fixed time-period: they are only revealed by quantitative analysis of the distribution of individual intervals.

\subsection{Values of the underlying parameters}

Best-fit values of $\mu$ and $\sigma$ were estimated for all participants and conditions. Figure 3 shows the average values of the parameters broken down by type of response and category of participant (controls and manifest). When comparing the three different tasks for the HD participants, $\mu$ is significantly different between the alternation and pronating tasks $(p=0.0011)$ and between pronating and single ( $p=0.0024)$, and $\sigma$ is significantly different for pronating versus alternating $(p=0.045)$, but no other comparisons are significant at $p=0.05$. When comparing the patients with the controls, for all three tasks the patients show the expected reduction in $\mu$ (equivalent to increased time between taps), but because of the very small group sizes in this pilot study, the only difference seen in Fig. 3, that is significant at $p=0.05$ (unpaired $t$ test), is for $\sigma$ with single tapping $(p=0.036)$.

It is instructive to plot $\mu$ and $\sigma$ as a scatter plot for the two groups (Fig. 4). What is then seen is an apparent segregation between the manifest patients (M) and the controls; once again, it would obviously be desirable to have more participants than was available for this preliminary evaluation.

Finally, some idea of the test's predictive validity can be seen by looking at the correlation between the observed values of $\mu$ and $\sigma$ and a more general measure of the patients' neurological status, the total motor score (TMS). Regression analysis reveals $R$ values that are significant or near-significant for $\mu$ (Pearson: $p=0.063,0.008$ and 0.065 (Fig. 5) for alternation, pronation and single tapping, respectively), but not for $\sigma$. 
Fig. 5 Relation between $\mu$ and $\sigma$ in each of three types of test, and the total motor score, across all manifest HD subjects and controls (the latter are assigned a TMS score of zero)

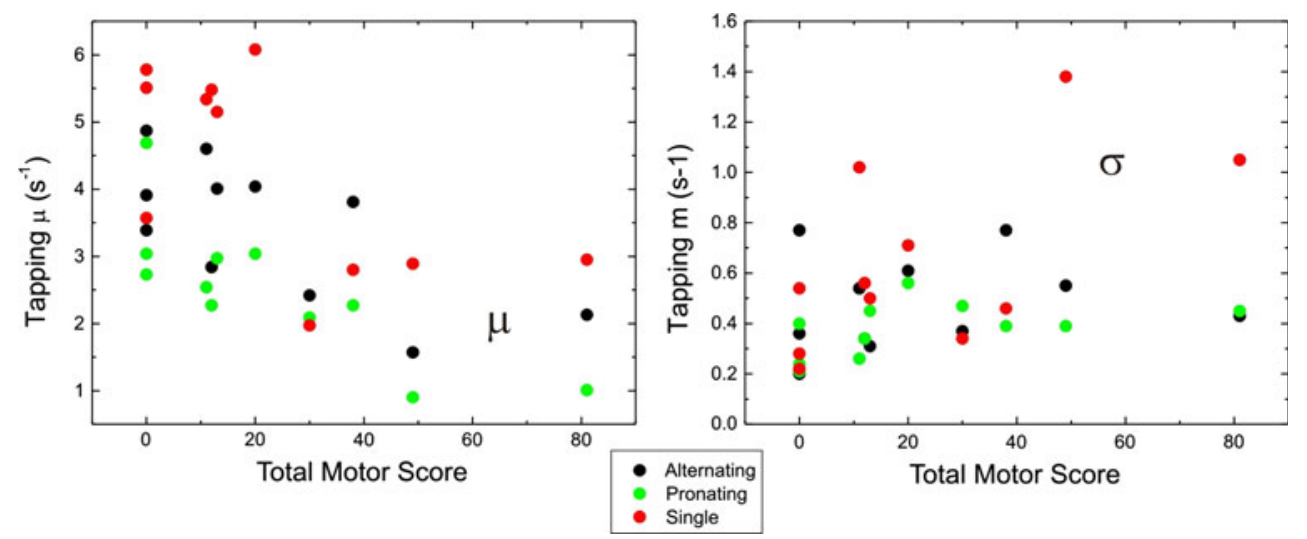

\section{Discussion}

The aim of this study was to evaluate the potential use of a new device for quantitative assessments in disorders, such as HD in which hand-tapping tapping is abnormal. It has two key features: first, the force required to be exerted by the subject to register a response is very low (essentially zero); this makes it feasible to use variations on the basic alternation task that can pose a greater challenge to a patient with relatively mild impairment. Figure $2 d$ provides a particularly clear example of this, when comparing ordinary alternation with the pronation task: the difference in $\mu$ for the two types of task is equivalent to over $450 \mathrm{~ms}$ of latency difference. The second potential benefit is that by providing sequential information about individual responses in each direction, a great deal of data are generated in a short period of time, from which much more can be calculated than the average response time that has previously been conventionally used. For simple tapping, the parameter $\sigma$ seems to provide particularly clear discrimination between subject groups (Fig. 3, right), and it is possible that a combination of $\mu$ and $\sigma$ together may be helpful in this respect (Fig. 4). Because responses in the two directions are not conflated, lateral asymmetries (Fig. 2c), suggesting a relatively one-sided functional impairment, become obvious and can be quantified. Other kinds of idiosyncrasies, not previously noted (such as the bimodality of Fig. 2a, or the sporadic inattention of Fig. 2b) are also revealed, and are equally capable of quantification. Another advantage of the device is that it is small, lightweight and portable and the software is easy-touse allowing its potential ease of introduction to a clinical environment.

This is, in other words, a simple tool that has the potential to address a general problem in studying not only in HD, but also other neurodegenerative disorders: a lack of objective and genuinely quantitative neurological tests, by which disease progress can be monitored and treatments evaluated with respect to the particular needs of individual patients. Obviously much more data are needed to provide true validation, and to discover what aspects of the interval distributions will be most useful for this process, and this work is currently in hand.

Acknowledgments This research was supported by a grant from the Wellcome Trust (073735), by the National Institute of Health Research (NIHR), by the Oxford Biomedical Research Centre, and by the Dementias and Neurodegenerative Diseases Research Network (DENDRON).

Open Access This article is distributed under the terms of the Creative Commons Attribution Noncommercial License which permits any noncommercial use, distribution, and reproduction in any medium, provided the original author(s) and source are credited.

\section{References}

1. Agostino R, Berardelli A, Formica A, Accornero N, Manfredi M (1992) Sequential arm movements in patients with Parkinson's disease. Huntington's disease and dystonia. Brain 115(Pt 5): 1481-1495

2. Andrich J, Saft C, Ostholt N, Muller T (2007) Assessment of simple movements and progression of Huntington's disease. J Neurol Neurosurg Psychiatry 78(4):405-407

3. Biglan KM, Ross CA, Langbehn DR, Aylward EH, Stout JC, Queller S et al (2009) Motor abnormalities in premanifest persons with Huntington's disease: The PREDICT-HD study. Mov Disord 24(12): 1763-1772

4. Carpenter RHS (1994) SPIC: a PC-based system for rapid measurement of saccadic responses. J Physiol 4P:480

5. Carpenter RH, Williams ML (1995) Neural computation of $\log$ likelihood in control of saccadic eye movements. Nature 377(6544):59-62

6. Fischer B, Ramsperger E (1984) Human express saccades: extremely short reaction times of goal directed eye movements. Exp Brain Res [Experimentelle Hirnforschung] 57(1):191-195

7. Garcia Ruiz PJ, Hernandez J, Cantarero S, Bartolome M, Sanchez Bernardos V, de Garcia Yebenez J (2002) Bradykinesia in Huntington's disease. A prospective, follow-up study. J Neurol 249(4):437-440

8. Hinton SC, Paulsen JS, Hoffmann RG, Reynolds NC, Zimbelman JL, Rao SM (2007) Motor timing variability increases in preclinical Huntington's disease patients as estimated onset of motor symptoms approaches. J Int Neuropsychol Soc 13(3):539-543 
9. Huntington's Disease Collaborative Research Group (1993) A novel gene containing a trinucleotide repeat that is expanded and unstable on Huntington's disease chromosomes. Cell 72:971983

10. Kirkwood SC, Siemers E, Bond C, Conneally PM, Christian JC, Foroud T (2000) Confirmation of subtle motor changes among presymptomatic carriers of the Huntington disease gene. Arch Neurol 57(7):1040-1044

11. Michell AW, Goodman AO, Silva AH, Lazic SE, Morton AJ, Barker RA (2008) Hand tapping: a simple, reproducible, objective marker of motor dysfunction in Huntington's disease. J Neurol 225(8):1145-1152

12. Muller T, Schafer S, Kuhn W, Przuntek H (2000) Correlation between tapping and inserting of pegs in Parkinson's disease. Can J Neurol Sci 27(4):311-315

13. Paulsen JS, Langbehn DR, Stout JC, Aylward E, Ross CA, Nance $M$ et al (2007) Detection of Huntington's disease decades before diagnosis: the Predict HD study. J Neurol Neurosurg Psychiatry 79(8):874-880

14. Paulsen JS, Hayden M, Stout JC, Langbehn DR, Aylward E, Ross CA et al (2006) Preparing for preventive clinical trials: the Predict-HD study. Arch Neurol 63(6):883-890

15. Saft C, Andrich J, Meisel NM, Przuntek H, Muller T (2006) Assessment of simple movements reflects impairment in Huntington's disease. Mov Disord 21(8):1208-1212

16. Tabrizi SJ, Langbehn DR, Leavitt BR, Roos RA, Durr A, Craufurd D et al (2009) Biological and clinical manifestations of Huntington's disease in the longitudinal TRACK-HD study: cross-sectional analysis of baseline data. Lancet Neurol 8(9):791-801

17. Thompson PD, Berardelli A, Rothwell JC, Day BL, Dick JP, Benecke R et al (1988) The coexistence of bradykinesia and chorea in Huntington's disease and its implications for theories of basal ganglia control of movement. Brain 111(Pt 2):223-244 